\title{
Phytosphingosine-1-phosphate and epidermal growth factor synergistically restore extracellular matrix in human dermal fibroblasts in vitro and in vivo
}

\author{
SEUNG BIN KWON ${ }^{1 *}$, SUNGKWAN AN $^{2 *}$, MIN JUNG KIM ${ }^{3}$, KA RAM KIM ${ }^{1}$, YOUNG MIN CHOI ${ }^{2}$, \\ KYU JOONG AHN ${ }^{3}$, IN-SOOK AN ${ }^{1}$ and HWA JUN CHA ${ }^{2}$ \\ ${ }^{1}$ KISCS Incorporated, Cheongju-si, Chungcheongbuk-do 361-951; ${ }^{2}$ Korea Institute for Skin and Clinical Sciences, \\ Konkuk University; ${ }^{3}$ Department of Dermatology, Konkuk University School of Medicine, \\ Seoul 143-701, Republic of Korea
}

Received December 11, 2015; Accepted December 23, 2016

DOI: $10.3892 / \mathrm{ijmm} .2017 .2866$

\begin{abstract}
Phytosphingosine-1-phosphate (PhS1P), which is found in plants and fungi, is generated by the phosphorylation of phytosphingosine and is structurally similar to molecules that promote cellular growth and proliferation. The aim of this study was to ascertain whether PhS1P displays synergistic effects together with epidermal growth factor (EGF), which is also critical for activating proliferation, migration and survival pathways. We utilized cultured human dermal fibroblasts (HDFs) and a number of assays, including western blotting, cell migration assays, quantitative (real-time) PCR, and viability assays. We found that PhS1P promoted the activity of EGF in vitro. We then conducted a clinical trial in females over 35 years of age, with visible signs of skin aging. By evaluating skin hydration, dermal density and thickness, length of fine wrinkles, and skin elasticity, we verified the clinical efficacy of a combined treatment of PhS1P and EGF in vivo. On the whole, our data suggest that PhS1P displays a synergistic anti-aging effect together with EGF, both in vitro and in vivo.
\end{abstract}

\section{Introduction}

The skin is composed of two layers: the epidermis and the dermis. Mesenchymal cells, known as dermal fibroblasts, reside within the dermis and generate the major components of the extracellular matrix (ECM), which organizes the dermal

Correspondence to: Dr Hwa Jun Cha, Korea Institute for Skin and Clinical Sciences, Konkuk University, 120 Neungdong-ro, Gwangjin-gu, Seoul 143-701, Republic of Korea

E-mail: hjcha@konkuk.ac.kr

*Contributed equally

Key words: phytosphingosine-1-phosphate, fibroblasts, aging, collagen, epidermal growth factor, AKT layer (1-3). Dermal fibroblasts have been reported to play a key role in controlling skin physiology, and their proliferation is also crucial for skin structural homeostasis and wound healing (4-6).

The epidermal growth factor receptor (EGFR), a glycoprotein on the cell surface membrane, acts as a signaling initiator. EGFR is composed of an extracellular receptor domain, an intracellular domain, and a transmembrane region. The intracellular domain acts as a tyrosine kinase, and activated EGFR initiates signal transduction pathways that regulate cellular proliferation, differentiation and survival $(7,8)$. EGFR is bound by epidermal growth factor (EGF), a small peptide, which stimulates EGFR to induce cell proliferation and migration $(2,8,9)$.

As a cellular signal transduction molecule, phytosphingosine-1-phosphate (PhS1P) is not abundant in animals. However, previous studies suggest that it is highly expressed in plants and fungi (3). PhS1P is generated by sphingosine kinase through phosphorylation of phytosphingosine. PhS1P has been shown to have a high affinity for the sphingosine-1-phosphate (S1P) receptor and shows structural similarity to S1P (3).

The aim of the present study was to ascertain whether PhS1P and EGF display synergistic effects, using in vitro assays and an in vivo clinical trial. Our results demonstrated that treatment with PhS1P and EGF induced an anti-aging effect in cell culture, as well as in study subjects, further suggesting that $\mathrm{PhS1P}$ holds promise as a potential anti-aging cosmetic ingredient.

\section{Materials and methods}

Cell culture. Human dermal fibroblasts (HDFs; Lonza, Basel, Switzerland) were cultured in Dulbecco's modified Eagle's medium (DMEM; Gibco/Life Technologies, Carlsbad, CA, USA), supplemented with $10 \%$ fetal bovine serum (FBS; Sigma-Aldrich, St. Louis, MO, USA) and $1 \%$ penicillin/streptomycin (Gibco/Life Technologies) at $37^{\circ} \mathrm{C}$ in an atmosphere of $5 \% \mathrm{CO}_{2}$. PhS1P (Avanti Polar Lipids, Alabaster, AL, USA) and EGF (R\&D Systems, Minneapolis, MN, USA) were dissolved in dimethyl sulfoxide (DMSO). 
Cell viability assay. HDFs were seeded at a density of $3 \times 10^{3}$ cells/well in 96-well plates and incubated for $24 \mathrm{~h}$. The cells were then either left untreated, pretreated with a range of $\mathrm{PhS1P}$ concentrations $(0-5 \mu \mathrm{M})$, or pretreated with both $\mathrm{PhS} 1 \mathrm{P}$ $(5 \mu \mathrm{M})$ and EGF $(10 \mathrm{ng} / \mathrm{ml})$ for $3 \mathrm{~h}$, prior to irradiation with UVB $\left(50 \mathrm{~mJ} / \mathrm{cm}^{2}\right)$. UVB irradiated cells were incubated for $24 \mathrm{~h}$, and cytotoxicity was evaluated using the 3-(4,5-dimethylthiazol-2-yl)-2,5-diphenyltetrazolium bromide (MTT) assay. Briefly, MTT tetrazolium salt $(0.5 \mathrm{mg} / \mathrm{ml}$; Sigma-Aldrich) was added to the cells for $4 \mathrm{~h}$. The medium was then replaced with DMSO, and the absorbance of each sample was measured at $595 \mathrm{~nm}$ using a plate reader (Bio-Rad Laboratories, Hercules, CA, USA).

Western blotting. Western blotting was performed as described in our previous study (2). Cells were collected and washed with cold phosphate-buffered saline (PBS). The cell pellets were lysed using modified radioimmunoprecipitation assay (RIPA) buffer [50 mM Tris, $\mathrm{pH} 8.0,150 \mathrm{mM} \mathrm{NaCl}, 1 \%$ NP-40, 0.5\% deoxycholate, $0.1 \%$ sodium dodecyl sulfate (SDS)] containing protease inhibitors (Complete Tablets, Mini, EDTA-free, EASYpack; Roche Applied Science, Mannheim, Germany) at $4^{\circ} \mathrm{C}$ for $20 \mathrm{~min}$. The lysates were then centrifuged at $12,000 \mathrm{x} \mathrm{g}$ for $30 \mathrm{~min}$, and the supernatant was decanted and saved. The concentration of total protein was determined using the Bradford assay (Bio-Rad Laboratories), and $50 \mu \mathrm{g}$ of total cellular protein was solubilized in SDS sample buffer and resolved by SDS-PAGE. Anti-ERK (M5670), anti-p-ERK (M9692), anti-AKT (SAB4500797) and anti-p-AKT (SAB4504332) primary antibodies for immunoblotting were purchased from Sigma-Aldrich.

Isolation of total RNA and quantitative (real-time) polymerase chain reaction ( $q P C R)$. Total RNA was isolated using TRIzol reagent (Invitrogen, Carlsbad, CA, USA) as previously described (10), and RNA purity and concentration were evaluated using MaestroNano ${ }^{\circledR}$ microspectrophotometer (Maestrogen, Las Vegas, NV, USA). Equal amounts of RNA were used for cDNA synthesis, and this was then used as a PCR template to examine the expression of EGFR, matrix metalloproteinase 1 (MMP1), and COL1A1. PCR primers were designed by Primer3 software (http://frodo.wi.mit.edu) (EGFR forward, 5'-CAGCG CTACCTTGTCATTCA-3' and reverse, 5'-TGCACTCAGAGA CCTCAGGA-3'; MMP1 forward, 5'-GGTCTCTGAGGGTC AAGCAG-3' and reverse, 5'-AGTTCATGGCTGCAACACG-3'; $\beta$-actin forward, 5'-GGATTCCTATGTGGGCGACGA-3' and reverse, 5'-CGCTCGGTGAGGATCTTCATG-3'); and qPCR was performed using EvaGreen dye (Solis BioDyne, Tartu, Estonia) and Line-Gene K software (Bioer, Hangzhou, China). The $\mathrm{Ct}$ value for each gene was normalized to that of $\beta$-actin.

Subjects for clinical evaluation. The study protocol was approved by the Institutional Review Board of the Korea Institute for Skin and Clinical Sciences (KISCS) Incorporated. Forty women over the age of 35 were registered in a randomized, double-blind clinical trial (control group: 43.85 \pm 5.07 years; experimental group: $46.40 \pm 5.75$ years). The subjects were selected based on age and the absence of skin conditions other than those determined to be age-related; none were pregnant or nursing. All subjects were informed about the objective of the study, provided signed informed consent, and agreed to use only study-associated products for skin care during the duration of the study. Exclusion criteria included itching, erythema, or excessive drinking or smoking. Subjects were divided into a control and an experimental group consisting of 20 subjects each. All conditions were identical for both groups, other than the exposure of the experimental group to the test material. The study lasted for 6 weeks, with none of the study subjects dropping out. Clinical parameters were evaluated three times, namely, before use, and after 3 and 6 weeks of use. At each evaluation, the investigator asked subjects about the condition of their skin and performed a visual examination of their skin condition, to assess any erythema, itching, scaling, edema, tingling, and burning sensation. The cream given to the experimental group contained 30 ppm PhS1P and 2 ppm EGF, whereas the cream given to the control group was prepared using the same volume of water in place of PhS1P and EGF.

Experimental procedures. To investigate the improvement in skin barrier function, subjects were instructed to apply $2 \mathrm{~g}$ of the test material to the face every morning and night for 4 weeks. Subjects and investigators were blinded as to which group received the experimental and control treatments. Moisture and transepidermal water loss (TEWL) were measured on the right cheek, and skin texture was evaluated on the left side of the forehead. All subjects washed with the cleanser provided and lay quietly in a room with constant temperature $\left(22 \pm 1^{\circ} \mathrm{C}\right)$ and humidity $(45 \pm 5 \%)$ before assessments were performed, so that they all would be evaluated under the same conditions.

Evaluation of skin moisture. To evaluate improvement in skin moisture, the DermaLab USB moisture probe (Cortex Technology, Inc., Hadsund, Denmark) was applied, and data were analyzed using the associated application software, version 1.09. All subjects were measured on the same region of the right cheek, five consecutive times, and the mean, maximum, and minimum values were determined. Measurements were taken three times, namely, before application, and after 3 and 6 weeks of use. The device applies the conductance measurement principle to measure the water-binding capacity of the stratum corneum; this conductance value correlates with skin moisture and is expressed in microsiemens $(\mu \mathrm{S})$.

Measurement of dermal density and thickness. To evaluate dermal density, a DUB skin scanner (tpm taberna pro medicum, Lueneburg, Germany) was used. Dermal density was measured $3 \mathrm{~cm}$ from the left eye, applying the couplant for ultrasonic examination. The analysis range was limited to the area between the dermis and upper panniculus. The measurements were taken three times, namely, before application, and after 3 and 6 weeks of use.

Measurement of length and evenness of crow's feet. Clinical images for the measurement of facial skin evenness were obtained by a PRIMOS Lite (field of view 45x30; GFMesstechnik GmbH, Teltow, Germany), and captured images were analyzed using the associated imaging software, PRIMOS Lite, version 5.6E. Three consecutive clinical images of the subject's crow's feet were captured. Facial skin roughness was assessed based on the Ra value, which is the average of all heights and depths relative to the reference plane. The $\mathrm{Ra}$ value is the most widely 

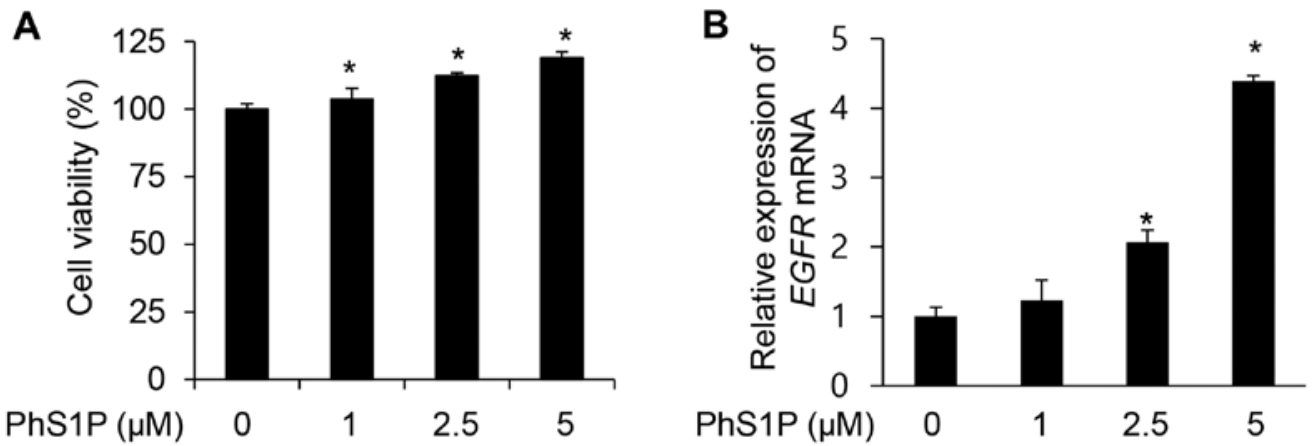

Figure 1. Cell viability and expression of epidermal growth factor receptor (EGFR) mRNA in human dermal fibroblasts (HDFs) treated with phytosphingosine1-phosphate (PhS1P). (A) Cell viability of HDFs treated with increasing amounts of PhS1P. (B) The relative mRNA expression of EGFR in PhS1P-treated HDFs. ${ }^{*} \mathrm{P}<0.01$ compared with non-treated HDFs, as determined by the Student's t-test.

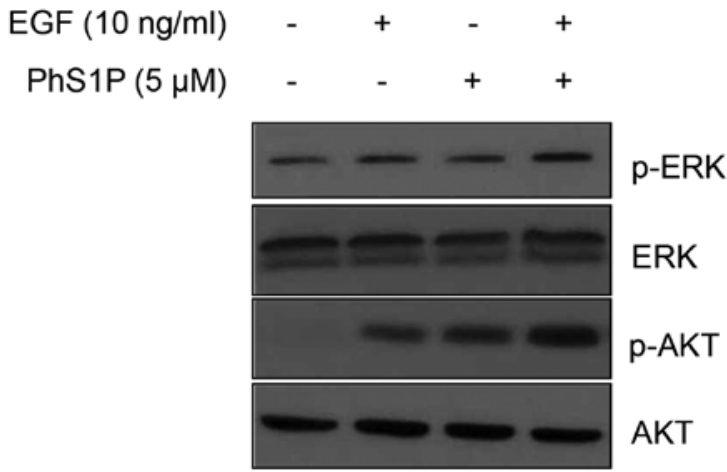

Figure 2. Effect of phytosphingosine-1-phosphate (PhS1P) on epidermal growth factor (EGF)-induced p-ERK, ERK, p-AKT and AKT protein expression in human dermal fibroblasts (HDFs).

used parameter of facial skin roughness and is the arithmetic mean of the maximum values of all measurements. To evaluate improvement in wrinkles, especially at the eye rim, a Robo skin analyzer CS50 (Inforward Inc., Tokyo, Japan) was used. The facial images were captured from each subject under identical positions, and with equal lighting, on the front, left, and right sides of the face. To evaluate improvement, measurements were taken three times, namely, before application, and after 3 and 6 weeks of use. We analyzed the captured images, matching the facial feature points accurately, and the unit of measurement for crow's feet length was mm.

Measurement of skin elasticity. To evaluate improvement in skin elasticity, a DermaLab USB elasticity probe (Cortex Technology, Inc.) was applied, and the results were analyzed using the associated application software, version 1.09. Measurements were obtained using a fixed elasticity probe on the left cheek of each subject. To analyze the elasticity measurements, Young's modulus (E) was calculated as a dosedependent representation of skin elasticity. The measurements of elasticity were taken three times, namely, before application, and after 3 and 6 weeks of use. The unit of measurement was MPa.

Statistical and mathematical analysis. In the cellular efficacy tests, all results are presented as the mean percentage \pm standard deviation (SD) of three independent experiments.
Differences with a P-value $<0.05$ or 0.001 , as determined by Student's t-test, were considered statistically significant. In clinical efficacy tests, statistical analyses were conducted using SPSS software (SPSS, version 17.0 for Windows; IBM, Armonk, NY, USA). Paired t-tests were performed in cases of repeated measurements on the same subject. To analyze subject questionnaires, the mean values, standard deviation, and percentages were used. The formula used to measure the percent change for each skin parameter was 'Percent change $=[(\mathrm{A}-\mathrm{B}) / \mathrm{B}] \mathrm{x} 100$ ', where $\mathrm{A}$ is defined as the individual value of any parameter at the 3 - and 6-week visits, and $\mathrm{B}$ represents the zero hour of the assessed parameter.

\section{Results and Discussion}

PhS1P induces HDF proliferation and acts as a cognate ligand for EGFR. The PhS1P molecule is structurally similar to $\mathrm{S} 1 \mathrm{P}$, suggesting they perform similar functions. S1P is known as a signal transduction molecule that induces cellular proliferation (11). We therefore, aimed to determine whether $\mathrm{PhS1P}$ would function similarly to induce the proliferation of HDFs. We first assessed the cytotoxicity of this molecule by treating HDFs with various PhS1P concentrations, ranging from 0 to $5 \mu \mathrm{M}$, for $24 \mathrm{~h}$. Cell viability was then measured using the MTT assay. Importantly, we observed that cell viability increased gradually with the increased dosage of PhS1P, up to a $23 \%$ increase at $5 \mu \mathrm{M} \mathrm{PhS1P}$ (Fig. 1A), suggesting that $\mathrm{PhS1P}$ induced HDF proliferation. Subsequently, in order to determine whether PhS1P affects EGFR expression, EGFR mRNA levels were measured in cells treated with PhS1P using qPCR. We observed that the relative expression of EGFR mRNA was increased 2-fold when cells were treated with $2.5 \mu \mathrm{M} \mathrm{PhS1P}$ and 4.5-fold when they were treated with $5 \mu \mathrm{M}$ $\mathrm{PhS1P}$ (Fig. 1B). These results revealed that PhS1P induced EGFR mRNA expression in HDFs, and demonstrated that this molecule has properties that suggest it may act as a ligand for EGFR.

Co-treatment with PhSIP and EGF induces phosphorylation of ERK and AKT in HDFs. The binding of EGF to EGFR activates downstream signaling proteins, such as ERK and AKT, by phosphorylation (12). Furthermore, S1P, a molecule that has structural and functional similarity to $\mathrm{PhS1P}$, also induces cellular proliferation and growth through the ERK 
A

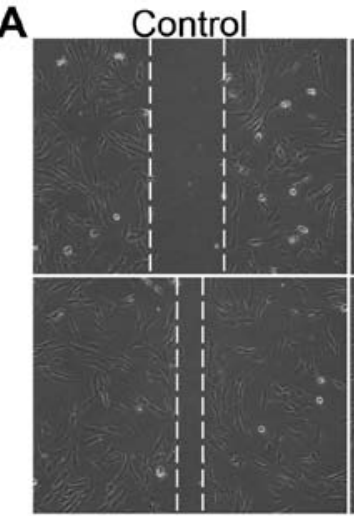

EGF

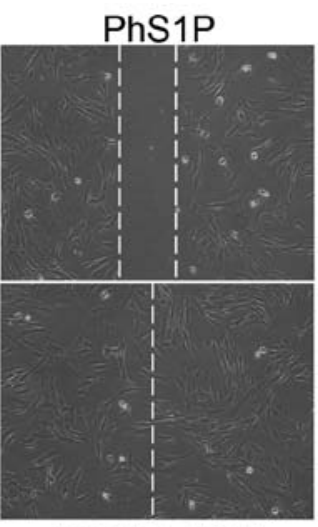

PhS1P+EGF

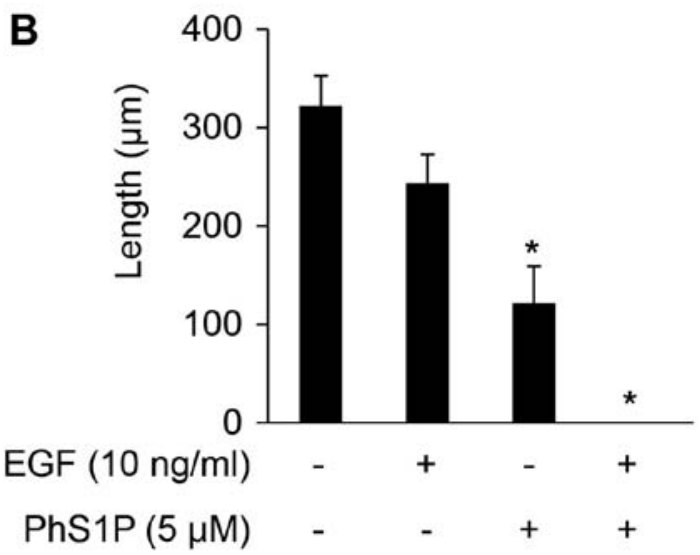

Figure 3. Cell migration of human dermal fibroblasts (HDFs) in a scratch wound healing assay after treatment with phytosphingosine-1-phosphate (PhS1P) and epidermal growth factor (EGF). (A) The microscopic images illustrate the migration of HDFs after treatment with PhS1P and/or EGF. (B) Average wound distance of scratched HDFs from quantitative analysis of the microscopic images. "P<0.01 compared with non-treated HDFs, as determined by the Student's t-test.

A

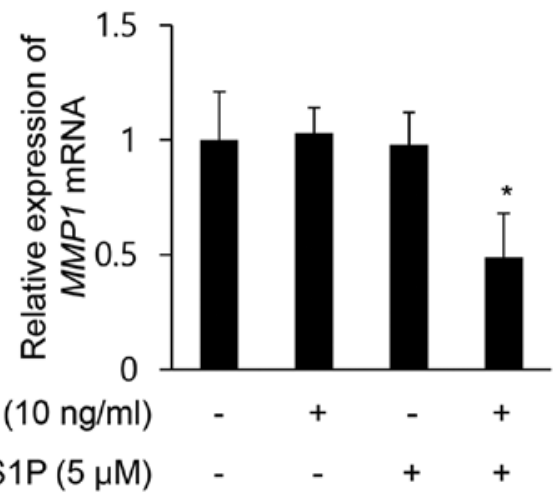

B

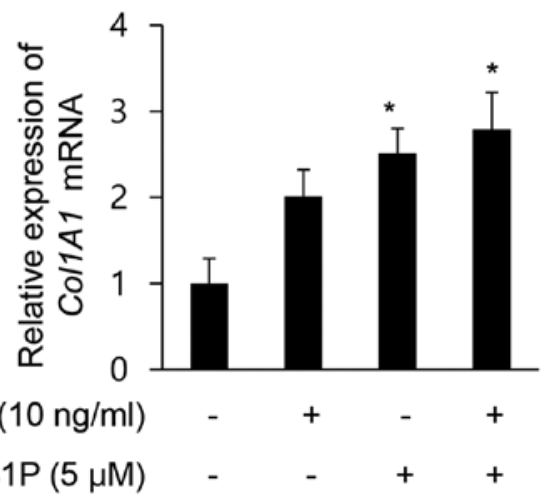

Figure 4. Effects of phytosphingosine-1-phosphate (PhS1P) and epidermal growth factor (EGF) on extracellular matrix (ECM)-regulated mRNA expression in human dermal fibroblasts (HDFs). (A) The relative levels of MMP1 mRNA in HDFs after treatment with EGF and PhS1P. (B) The relative levels of COL1A1 mRNA in HDFs after treatment with EGF and PhS1P. $\mathrm{P}<0.01$ compared with non-treated HDFs, as determined by the Student's t-test.

and AKT pathways (13), suggesting that $\mathrm{PhS1P}$ may have a similar function. To test this, HDFs were treated with $\mathrm{PhS1P}$ $(5 \mu \mathrm{M})$ and EGF (10 $\mathrm{ng} / \mathrm{ml})$, alone and in combination, and the protein levels of ERK, phospho-ERK (p-ERK), AKT, and phospho-AKT (p-AKT) were measured using western blotting. Treatment with $10 \mathrm{ng} / \mathrm{ml}$ EGF or $5 \mu \mathrm{M}$ PhS1P induced phosphorylation of ERK and AKT (Fig. 2). Importantly, in cells co-treated with PhS1P and EGF, the p-AKT and p-ERK levels were significantly higher than these levels in the cells treated with either molecule alone. These results suggest that $\mathrm{PhS1P}$ exerts a synergistic effect on EGF-induced expression of p-ERK and p-AKT, and may function to induce cellular proliferation through these pathways.

Treatment with PhSIP and EGF promotes wound healing in a scratch plate assay. Both the proliferation and migration of HDFs are essential for cutaneous wound healing (14), and treatment with a combination of EGF and EGFR activates the AKT and ERK signaling pathways, inducing cellular migration $(12,15)$. Here, we observed that co-treatment with EGF and $\mathrm{PhS1P}$ also significantly increased AKT and ERK phosphorylation in HDFs (Fig. 2). In order to determine whether the enhanced phosphorylation of AKT and ERK observed in cells treated with EGF and PhS1P affects HDF migra- tion, we utilized a scratch wound healing assay. As shown in Fig. 3A and B, the wound distance was decreased in the HDFs treated with either PhS1P or EGF. Additionally, when cells were co-treated with $\mathrm{PhS1P}$ and EGF, the wound distance decreased further, as compared to the HDFs treated with either molecule alone (Fig. 3A and B). These results demonstrated that PhS1P induced cellular migration in HDFs, and did so more efficiently in combination with EGF.

PhS1P promotes EGF-induced ECM-related gene expression. Fibroblasts can alter the ECM by degrading fibrin and promoting the production of collagen $(16,17)$ and components of the ECM, particularly collagen I and III, are essential for wound healing $(17,18)$. Collagen is the major structural component of dermal tissue, and therefore, structural changes in this molecule result in typical signs of aging, such as wrinkling $(19,20)$. One member of the MMP family, MMP1 (collagenase-1), is generated in fibroblasts and is also capable of degrading type I and III fibrillar collagen (21). Members of the MMP family have also been shown to be capable of degrading all components of the ECM, including collagen, elastin, laminin, proteoglycans, and fibronectin $(21,22)$. To investigate the effects of PhS1P on the expression of MMP1 and COL1A1, qPCR was performed on HDFs treated with 


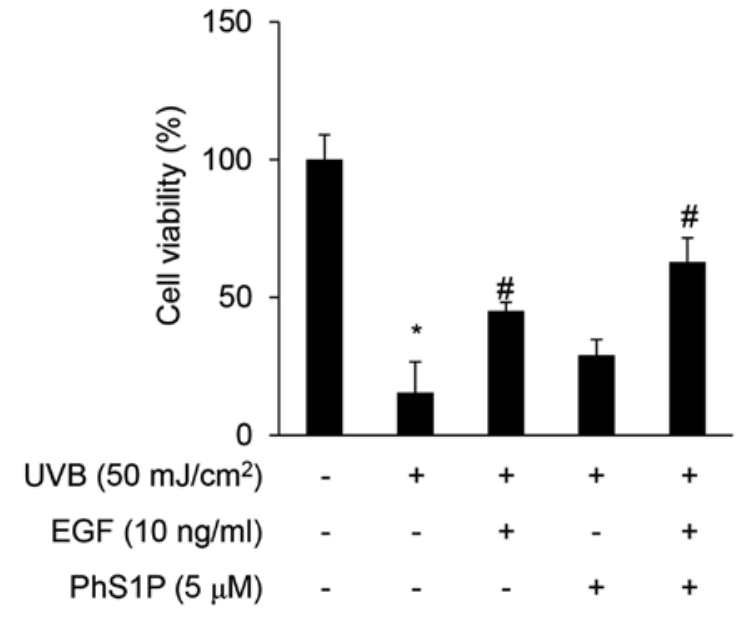

Figure 5. Phytosphingosine-1-phosphate (PhS1P) and epidermal growth factor (EGF) protect against UVB-induced cell death in irradiated human dermal fibroblasts (HDFs). ${ }^{*} \mathrm{P}<0.01$ compared with non-treated HDFs and ${ }^{\#} \mathrm{P}<0.01$ compared with UVB-irradiated HDFs, as determined by the Student's t-test.

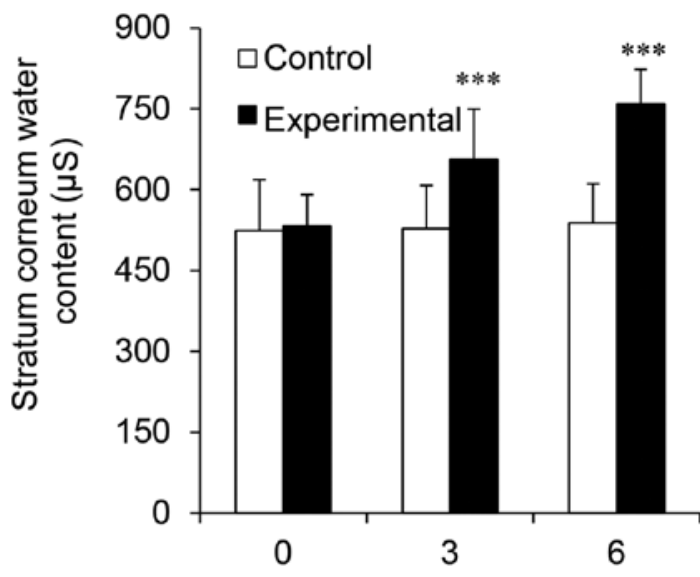

(Weeks)

Figure 6. Effects of a phytosphingosine-1-phosphate (PhS1P) and epidermal growth factor (EGF)-containing cream on skin moisture content in study subjects. Mean stratum corneum water content measurements in experimental and control subjects at 0,3 and 6 weeks. ${ }^{* * *} \mathrm{P}<0.001$ compared with control group, as determined by the Student's t-test.
EGF $(10 \mathrm{ng} / \mathrm{ml})$ and/or PhS1P $(5 \mu \mathrm{M})$. We observed that the relative expression of MMP1 decreased 0.49-fold in the HDFs treated with both PhS1P and EGF (Fig. 4A). Conversely, treatment with both molecules increased COL1A1 mRNA levels 2.79-fold (Fig. 4B). These results demonstrated that PhS1P promoted EGF-induced COL1A1 mRNA expression, and together, these molecules suppressed MMP1 expression, two genes that regulate the ECM and influence skin elasticity.

PhSIP protects against UVB-induced decreased cell viability and stimulates EGF-induced cell viability. Ultraviolet (UV) radiation is a well-characterized mutagen that induces both skin aging and cancer. Here, we showed that PhS1P stimulated cell migration and promoted the expression of cellular proliferation markers. We, therefore, aimed to determine whether PhS1P can protect HDFs from UVB-induced damage. HDFs were irradiated with UVB at $50 \mathrm{~mJ} / \mathrm{cm}^{2}$, and under these conditions, only $15.37 \%$ of the cells remained viable. However, the cells treated with either EGF or PhS1P prior to UVB irradiation retained a viability of 45.12 and $28.73 \%$, respectively. Notably, HDFs co-treated with both PhS1P and EGF before UVB irradiation maintained a cell viability of $62.78 \%$ (Fig. 5). These data suggest that $\mathrm{PhS1P}$ protects against UVB-induced decreased cell viability, and further indicate that its protective ability is increased in the presence of EGF.

PhS1P improves skin moisture content. Collectively, our in vitro data suggest that $\mathrm{PhS1P}$, in combination with EGF, can promote pathways that are protective against aging-associated skin damage. We therefore, designed a double-blind clinical trial to test the efficacy of a cream containing PhS1P and EGF in women $>35$ years of age. Skin dryness is one of the hallmarks of skin aging (23), and therefore, we analyzed skin moisture content in the study subjects using the DermaLab USB moisture probe. We observed that in the control group, which used a cream that did not contain either PhS1P or EGF, the skin conductance (which directly reflects moisture content) was $523.93 \mu \mathrm{S}$ at the beginning of the study, and $527.45 \mu \mathrm{S}$ and $538.09 \mu \mathrm{S}$ after 3 and 6 weeks of applica- tion, respectively (Fig. 6). We then calculated the degree of improvement (Fig. 6) and found that the skin conductance of the control group increased by 0.67 and $2.70 \%$ after 3 and 6 weeks, respectively. These changes were not statistically significant $(P>0.05)$, indicating that the control cream had no measurable effect on moisture content. Conversely, the skin conductance in the experimental group, which used the $\mathrm{PhS1P}$ and EGF-containing cream, was $532.37 \mu \mathrm{S}$ before use, $655.95 \mu \mathrm{S}$ after 3 weeks, and $758.88 \mu \mathrm{S}$ after 6 weeks (Fig. 6). Notably, the use of the PhS1P and EGF-containing cream significantly improved skin conductance by 23.21 and $42.55 \%$ after 3 and 6 weeks, respectively $(\mathrm{P}<0.001)$. These experiments demonstrated that the use of the PhS1P and EGF-containing cream improved skin moisture content in the study subjects.

PhSIP improves EGF-induced dermal thickness and density. During the aging process, dermal thickness and density are reduced over time. Concurrently, MMP proteins show increased expression and degrade skin substrate proteins (24). Clinically, it has been shown that dermal thickness and density decrease with increasing concentrations of MMPs, as these proteins degrade albuminoids and the collagen layer (24). Here, we showed that PhS1P and EGF downregulated MMP1 and promoted expression of the collagen-encoding gene, COL1A1. We, therefore, evaluated the synergistic efficacy of $\mathrm{PhS1P}$ and EFG on dermal thickness and density in aging skin in vivo. In the control subjects, dermal thickness measurements were $94.00 \mu \mathrm{m}$ before use, and 96.85 and $94.75 \mu \mathrm{m}$ after 3 and 6 weeks of use, respectively. Thickness measurements in the subjects using the PhS1P and EGF-containing cream were $115.00 \mu \mathrm{m}$ before use, and 125.45 and $147.15 \mu \mathrm{m}$ after 3 and 6 weeks of application, respectively; these data were statistically significant $(\mathrm{P}<0.001)$. We then calculated the improvement in dermal thickness as a percentage, based on the values before and after application. Notably, the dermal thickness improvement was $3.03 \%$ after 3 weeks and $0.80 \%$ after 6 weeks in the control group, whereas the experimental group showed improvements in dermal thickness of 9.09 and $27.96 \%$ after 3 and 6 weeks of use, respectively (Fig. 7A). 

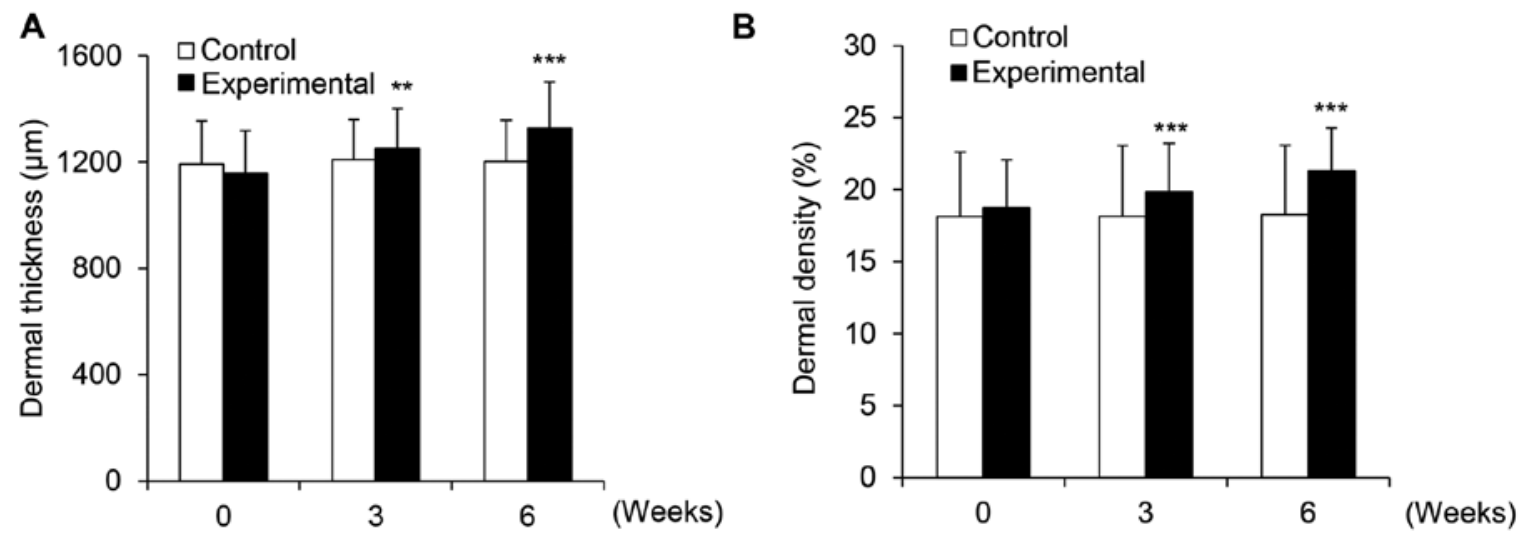

Figure 7. Effects of the PhS1P and epidermal growth factor (EGF)-containing cream on dermal thickness and density. (A) Mean dermal thickness measurements at 0,3 and 6 weeks. (B) Mean dermal density measurements at 0,3 and 6 weeks. ${ }^{* *} \mathrm{P}<0.01$ and ${ }^{* * * *} \mathrm{P}<0.001$ compared with control group, as determined by the Student's t-test.
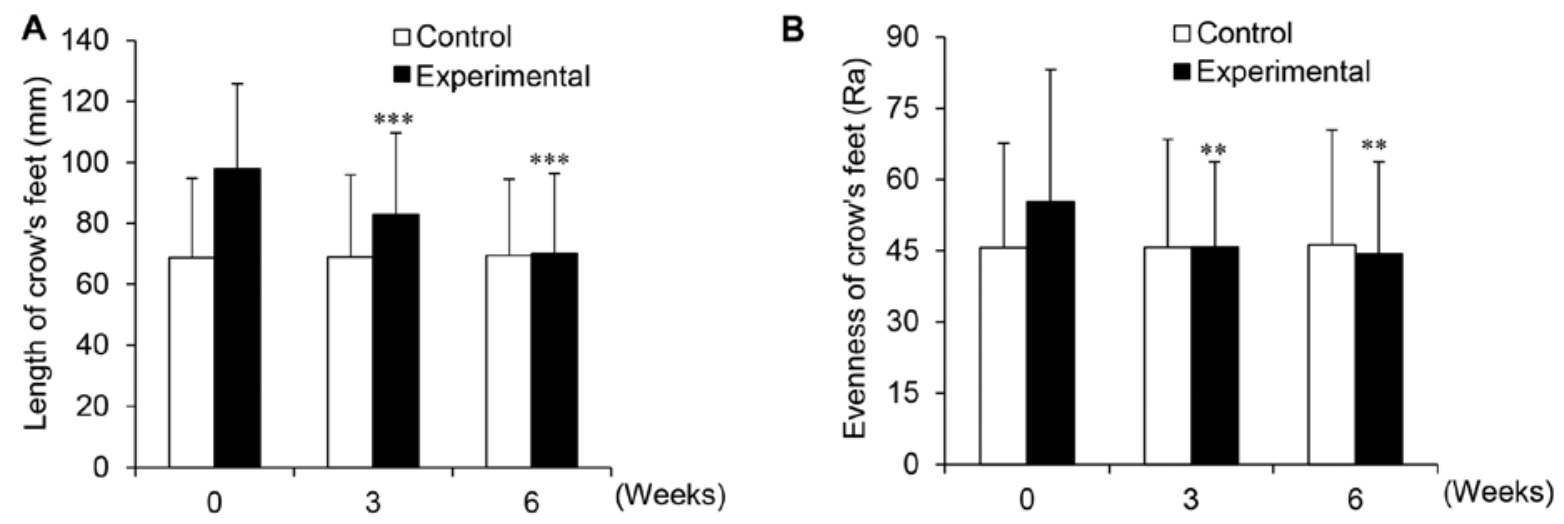

Figure 8. Effects of the PhS1P and epidermal growth factor (EGF)-containing cream on crow's feet. (A) The mean length of crow's feet at 0,3 and 6 weeks. (B) The mean crow's feet evenness values at 0,3 and 6 weeks. ${ }^{* *} \mathrm{P}<0.01$ and ${ }^{* * * *} \mathrm{P}<0.001$ compared with control group, as determined by the Student's t-test.

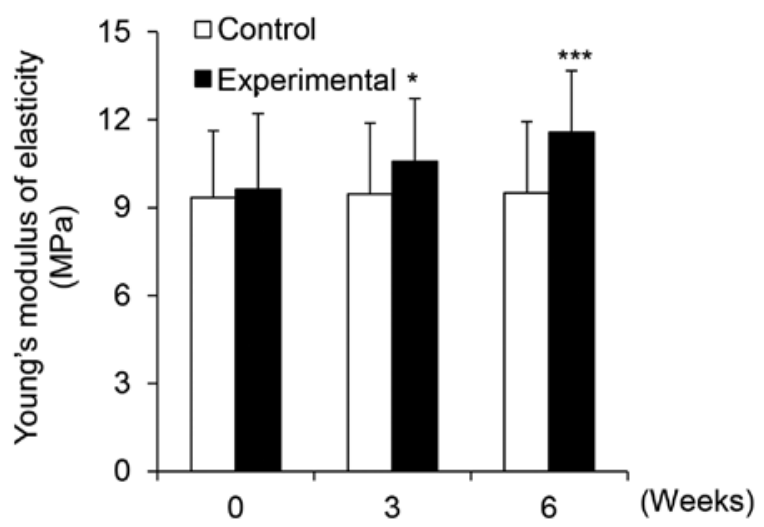

Figure 9. Effects of the PhS1P and epidermal growth factor (EGF)-containing cream on skin elasticity. ${ }^{*} \mathrm{P}<0.05$ and ${ }^{* * * *} \mathrm{P}<0.001$ compared with control group, as determined by the Student's t-test.

Dermal density measurements are represented as percentages, which are proportional to the skin density. In the subjects using the control cream, density measurements were $18.13 \%$ before application, and 18.16 and $18.28 \%$ after 3 and 6 weeks of use, respectively. Conversely, the subjects using the experimental cream displayed an average density of $18.76 \%$ before application, and densities of 19.85 and $21.30 \%$ after 3 and 6 weeks of use, respectively (Fig. 7B). The results for the experimental group were statistically significant $(\mathrm{P}<0.001)$. We then calculated the improvement in dermal density and found that application of the experimental cream resulted in improvements of 5.82 and $13.57 \%$ after 3 and 6 weeks, respectively, whereas those using the control cream improved by only 0.20 and $0.84 \%$, respectively. These results demonstrated that PhS1P, in combination with EGF, improved both dermal thickness and density.

Cream containing PhSIP and EGF improves the length and evenness of crow's feet. Wrinkles occur in response to structural changes in cells and tissues, and arise due to intrinsic aging factors, including decreases in collagen and elasticity, denaturalization of elastic fibers and the stratum corneum, and loss of skin moisture (25). Wrinkles can also form as a result of extrinsic aging factors, such as reactive oxygen species (ROS), which damage lipids and proteins in the skin via inflammatory cytokine production $(26,27)$. We, therefore, determined whether the PhS1P and EGF-containing cream can relieve wrinkles through clinical efficacy experiments. As shown in Fig. 8A, in the control group, the mean length of crow's feet was $68.90 \mathrm{~mm}$ before application, and 68.90 and $69.45 \mathrm{~mm}$ after 3 and 6 weeks, respectively. Conversely, for the experimental group, the mean length was $97.75 \mathrm{~mm}$ before application, and 82.80 and $70.20 \mathrm{~mm}$ after 3 and 6 weeks application, respectively; these values were statistically significant $(\mathrm{P}<0.001)$. When improve- 
ment over time was calculated, for the experimental group, the length of crow's feet improved markedly by 15.29 and $28.18 \%$ after 3 and 6 weeks, respectively. In the control group, improvement was only $0.29 \%$ after 3 weeks and $1.09 \%$ after 6 weeks. Based on these results, we concluded that the cream containing both $\mathrm{PhS1P}$ and EGF decreased wrinkle length in vivo.

When we evaluated the evenness of crow's feet, we found that in the control group, this was $45.57 \mathrm{Ra}$ before application, and 45.66 and $46.27 \mathrm{Ra}$ after 3 and 6 weeks, respectively. However, in the experimental group, the evenness was 55.37 Ra before application, and 45.81 and 44.39 Ra after 3 and 6 weeks of application, respectively (Fig. 8B); these values were statistically significant $(\mathrm{P}<0.001)$. To compare improvement in crow's feet evenness between the control and experimental group, we calculated the percentage of improvement using the values before application, and after 3 and 6 weeks of use. The control group showed an improvement of $0.20 \%$ after 3 weeks and $1.54 \%$ after 6 weeks application, whereas for the experimental group, improvement was $17.27 \%$ after 3 weeks and $19.83 \%$ after 6 weeks of use. Based on these data, we concluded that the cream containing PhS1P and EGF improved facial wrinkles, particularly in regards to the length and evenness of the crow's feet.

PhSIP and EGF-containing cream improves skin elasticity. The dermis is composed of dermal fibroblasts that exist within the extracellular matrix consisting of fibrous proteins, and it is largely involved in the regulation of skin elasticity. Various factors cause wrinkle formation and decrease skin elasticity through the denaturation of dermal structural components, including collagen and elastin (28). Therefore, in this experiment, we investigated the effects of the PhS1P and EGF-containing cream on skin elasticity. Using a DermaLab USB, we measured elasticity in our study subjects and found that before application, the mean elasticity was 9.35 , whereas it was 9.46 and 9.51 after 3 and 6 weeks, respectively. For the experimental group, mean elasticity was 9.63 before application, and 10.58 and 11.57 after 3 and 6 weeks of application, respectively (Fig. 9); these values were statistically significant $(\mathrm{P}<0.001)$. When assessing improvement in skin elasticity over time, the control group improved 1.18 and $1.71 \%$ after 3 and 6 weeks application, respectively. Conversely, the experimental group showed an improvement of 9.81 and $20.15 \%$ after 3 and 6 weeks, respectively. From these data, we concluded that cream containing $\mathrm{PhS1P}$ and EGF significantly improved skin elasticity.

Analysis of adverse effects of the PhS1P and EGF-containing cream. In the present study, investigators asked the subjects individually regarding the condition of their skin and performed a visual evaluation to assess any possible skin reactions, such as erythema, itching, scaling, tingling, tightness, prickling and burning sensation at every visit. No unusual reactions were reported, based on either the visual evaluation or the questionnaire.

In the present study, we showed that $\mathrm{PhS1P}$ can act as a stimulator of EGFR, inducing signal transduction to promote cell proliferation and migration. Our in vitro experiments demonstrated that PhS1P upregulated EGFR mRNA expression and displayed a synergistic effects with EGF at the cellular level. Co-treatment of HDFs with PhS1P and EGF enhanced cellular migration, protected against UVB-induced decreased cell viability, and influenced both the phosphorylation and expression of molecules critical for regulating cellular proliferation and the ECM. Furthermore, our in vivo experiments demonstrated that application of the cream containing $\mathrm{PhS1P}$ and EGF improved skin hydration, dermal density and thickness, evenness of crow's feet, and skin elasticity. EGF is a well-known and highly valued cosmetic ingredient that has shown efficacy as an anti-aging agent (29). This study suggests that $\mathrm{PhS1P}$ may represent a new efficacious antiaging cosmetic ingredient, which displays synergistic effects with EGF in aged skin efficacy trials.

\section{Acknowledgements}

This study was supported by the KU Research Professor (H.-J.C.) Program of Konkuk University. Funding was also provided by a grant from the Ministry of Science, ICT and Future Planning (no. 20110028646) of the Republic of Korea and a grant from the Korean Health Technology R\&D Project, Ministry of Health and Welfare, Republic of Korea (grant no. HN13C0075).

\section{References}

1. Sorrell JM and Caplan AI: Fibroblast heterogeneity: More than skin deep. J Cell Sci 117: 667-675, 2004

2. Cha HJ, Lee JP, Lee KS, Lee KK, Choi MJ, Lee DK, Kim KN and An S: Phytosphigosine-1-phosphate increases sensitivity of EGF-dependent cell proliferation. Int J Mol Med 33: 649-653, 2014.

3. Lee JP, Cha HJ, Lee KS, Lee KK, Son JH, Kim KN, Lee DK and An S: Phytosphingosine-1-phosphate represses the hydrogen peroxide-induced activation of c-Jun N-terminal kinase in human dermal fibroblasts through the phosphatidylinositol 3-kinase/Akt pathway. Arch Dermatol Res 304: 673-678, 2012.

4. Crigler L, Kazhanie A, Yoon TJ, Zakhari J, Anders J, Taylor B and Virador VM: Isolation of a mesenchymal cell population from murine dermis that contains progenitors of multiple cell lineages. FASEB J 21: 2050-2063, 2007.

5. Giro MG, Oikarinen AI, Oikarinen H, Sephel G, Uitto J and Davidson JM: Demonstration of elastin gene expression in human skin fibroblast cultures and reduced tropoelastin production by cells from a patient with atrophoderma. J Clin Invest 75: 672-678, 1985.

6. Schreier T, Degen E and Baschong W: Fibroblast migration and proliferation during in vitro wound healing. A quantitative comparison between various growth factors and a low molecular weight blood dialysate used in the clinic to normalize impaired wound healing. Res Exp Med (Berl) 193: 195-205, 1993.

7. Herbst RS: Review of epidermal growth factor receptor biology. Int J Radiat Oncol Biol Phys 59 (Suppl 2): 21-26, 2004.

8. Carpenter G and Cohen S: Epidermal growth factor. J Biol Chem 265: 7709-7712, 1990.

9. Allen G: Cosmetics - chemical technology or biotechnology? Int J Cosmet Sci 6: 61-69, 1984.

10. Cha HJ, Lee OK, Kim SY, Ko JM, Kim SY, Son JH, Han HJ, Li S, Kim SY, Ahn KJ, et al: MicroRNA expression profiling of p-phenylenediamine treatment in human keratinocyte cell line. Mol Cell Toxicol 11: 19-28, 2015.

11. Kim MK, Park KS, Lee H, Kim YD, Yun J and Bae YS: Phytosphingosine-1-phosphate stimulates chemotactic migration of L2071 mouse fibroblasts via pertussis toxin-sensitive G-proteins. Exp Mol Med 39: 185-194, 2007.

12. Normanno N, De Luca A, Bianco C, Strizzi L, Mancino M, Maiello MR, Carotenuto A, De Feo G, Caponigro F and Salomon DS: Epidermal growth factor receptor (EGFR) signaling in cancer. Gene 366: 2-16, 2006.

13. Pan HY, Yang H, Shao MY, Xu J, Zhang P, Cheng R and Hu T: Sphingosine-1-phosphate mediates AKT/ERK maintenance of dental pulp homoeostasis. Int Endod J 48: 460-468, 2015. 
14. Stevenson S and Thornton J: Effect of estrogens on skin aging and the potential role of SERMs. Clin Interv Aging 2: 283-297, 2007.

15. Puccinelli TJ, Bertics PJ and Masters KS: Regulation of keratinocyte signaling and function via changes in epidermal growth factor presentation. Acta Biomater 6: 3415-3425, 2010.

16. Ivarsson M, McWhirter A, Borg TK and Rubin K: Type I collagen synthesis in cultured human fibroblasts: Regulation by cell spreading, platelet-derived growth factor and interactions with collagen fibers. Matrix Biol 16: 409-425, 1998.

17. McDougall S, Dallon J, Sherratt J and Maini P: Fibroblast migration and collagen deposition during dermal wound healing: Mathematical modelling and clinical implications. Philos Trans A Math Phys Eng Sci 364: 1385-1405, 2006.

18. Ho MT, Kang HS, Huh JS, Kim YM, Lim Y and Cho M: Effects of the novel compound DK223 ([1E,2E-1,2-Bis(6-methoxy$2 \mathrm{H}$-chromen-3-yl)methylene]hydrazine) on migration and proliferation of human keratinocytes and primary dermal fibroblasts. Int J Mol Sci 15: 13091-13110, 2014.

19. Kim HM, Lee DE, Park SD, Kim YT, Kim YJ, Jeong JW, Jang SS, Ahn YT, Sim JH, Huh CS, et al: Oral administration of Lactobacillus plantarum HY7714 protects hairless mouse against ultraviolet B-induced photoaging. J Microbiol Biotechnol 24 1583-1591, 2014.

20. Chung JH, Seo JY, Choi HR, Lee MK, Youn CS, Rhie G, Cho KH, Kim KH, Park KC and Eun HC: Modulation of skin collagen metabolism in aged and photoaged human skin in vivo. J Invest Dermatol 117: 1218-1224, 2001.

21. Cho S, Won CH, Lee DH, Lee MJ, Lee S, So SH, Lee SK, Koo BS, Kim NM and Chung JH: Red ginseng root extract mixed with Torilus fructus and Corni fructus improves facial wrinkles and increases type I procollagen synthesis in human skin: A randomized, double-blind, placebo-controlled study. J Med Food 12: 1252-1259, 2009.
22. Inomata S, Matsunaga Y, Amano S, Takada K, Kobayashi K, Tsunenaga M, Nishiyama T, Kohno Y and Fukuda M: Possible involvement of gelatinases in basement membrane damage and wrinkle formation in chronically ultraviolet B-exposed hairless mouse. J Invest Dermatol 120: 128-134, 2003.

23. Asserin J, Lati E, Shioya T and Prawitt J: The effect of oral collagen peptide supplementation on skin moisture and the dermal collagen network: Evidence from an ex vivo model and randomized, placebo-controlled clinical trials. J Cosmet Dermatol 14: 291-301; Epub ahead of print, 2015.

24. Nwomeh BC, Liang HX, Diegelmann RF, Cohen IK and Yager DR: Dynamics of the matrix metalloproteinases MMP-1 and MMP-8 in acute open human dermal wounds. Wound Repair Regen 6: 127-134, 1998.

25. Parrado J, Bougria M, Ayala A, Castaño A and Machado A: Effects of aging on the various steps of protein synthesis: Fragmentation of elongation factor 2. Free Radic Biol Med 26: 362-370, 1999.

26. Fisher GJ, Wang ZQ, Datta SC, Varani J, Kang S and Voorhees JJ: Pathophysiology of premature skin aging induced by ultraviolet light. N Engl J Med 337: 1419-1428, 1997.

27. Fisher GJ, Kang S, Varani J, Bata-Csorgo Z, Wan Y, Datta S and Voorhees JJ: Mechanisms of photoaging and chronological skin aging. Arch Dermatol 138: 1462-1470, 2002.

28. Kimura Y and Sumiyoshi M: Olive leaf extract and its main component oleuropein prevent chronic ultraviolet B radiationinduced skin damage and carcinogenesis in hairless mice. J Nutr 139: 2079-2086, 2009.

29. Schouest JM, Luu TK and Moy RL: Improved texture and appearance of human facial skin after daily topical application of barley produced, synthetic, human-like epidermal growth factor (EGF) serum. J Drugs Dermatol 11: 613-620, 2012. 